\title{
A NOTE ON INCLUSION PROPERTIES OF WEIGHTED ORLICZ SPACES
}

\author{
AL A. MASTA ${ }^{1}$, IFRONIKA ${ }^{2}$, AND MUHAMMAD TAQIYUDDIN ${ }^{3}$ \\ ${ }^{1,3}$ Department of Mathematics Education, \\ Universitas Pendidikan Indonesia, Bandung 40154, Indonesia \\ ${ }^{2}$ Faculty of Mathematics and Natural Sciences, \\ Institut Teknologi Bandung, Bandung 40132, Indonesia \\ 1alazhari.masta@upi.edu, ${ }^{2}$ ifronika@math.itb.ac.id, ${ }^{3}$ taqi94@hotmail.com
}

\begin{abstract}
In this paper we present sufficient and necessary conditions for the inclusion relation between two weighted Orlicz spaces which complete the Osançliol result in 2014. One of the keys to prove our results is to use the norm of the characteristic functions of the balls in $\mathbb{R}^{n}$.

Key words and phrases: Inclusion property, weighted Lebesgue spaces, weighted Orlicz spaces.
\end{abstract}

\section{INTRODUCTION}

Orlicz spaces are generalization of Lebesgue spaces which were firstly introduced by Z. W. Birnbaum and W. Orlicz in 1931 (see [5, 14]). Let us first recall the definition of Orlicz spaces. Let $\Phi:[0, \infty) \rightarrow[0, \infty)$ be a Young function [that is, $\Phi$ is convex, $\lim _{t \rightarrow 0} \Phi(t)=0=\Phi(0)$, left-continuous and $\left.\lim _{t \rightarrow \infty} \Phi(t)=\infty\right]$. The Orlicz space $L_{\Phi}\left(\mathbb{R}^{n}\right)$ is the set of measurable functions $f: \mathbb{R}^{n} \rightarrow \mathbb{R}$ such that $\int_{\mathbb{R}^{n}} \Phi(a|f(x)|) d x<\infty$ for some $a>0$. The space $L_{\Phi}\left(\mathbb{R}^{n}\right)$ is a Banach space equipped with the norm

$$
\|f\|_{L_{\Phi}\left(\mathbb{R}^{n}\right)}:=\inf \left\{b>0: \int_{\mathbb{R}^{n}} \Phi\left(\frac{|f(x)|}{b}\right) d x \leq 1\right\} .
$$

Meanwhile, for $\Phi$ is a Young function, the weak Orlicz space $w L_{\Phi}\left(\mathbb{R}^{n}\right)$ is the set of all measurable functions $f: \mathbb{R}^{n} \rightarrow \mathbb{R}$ such that

$$
\|f\|_{w L_{\Phi}\left(\mathbb{R}^{n}\right)}:=\inf \left\{b>0: \sup _{t>0} \Phi(t) \mu\left(\left\{x \in \mathbb{R}^{n}: \frac{|f(x)|}{b}>t\right\}\right) \leq 1\right\}<\infty .
$$

2020 Mathematics Subject Classification: 11097, 2182, 3170 Received: 08-11-2018, accepted: 19-12-2018. 
Now, we move to the weighted Orlicz spaces and weighted weak Orlicz spaces. Let $\Phi$ be a Young function and $u$ is a weight on $\mathbb{R}^{n}$ (i.e $u: \mathbb{R}^{n} \rightarrow(0, \infty)$ is a measurable function). The weighted Orlicz space $L_{\Phi}^{u}\left(\mathbb{R}^{n}\right)$ is the set of all measurable functions $f: \mathbb{R}^{n} \rightarrow \mathbb{R}$ such that uf $\in L_{\Phi}\left(\mathbb{R}^{n}\right)$. Note that, the space $L_{\Phi}^{u}\left(\mathbb{R}^{n}\right)$ is a Banach space equipped with the norm

$$
\|f\|_{L_{\Phi}^{u}\left(\mathbb{R}^{n}\right)}:=\|u f\|_{L_{\Phi}\left(\mathbb{R}^{n}\right)}=\inf \left\{b>0: \int_{\mathbb{R}^{n}} \Phi\left(\frac{|u(x) f(x)|}{b}\right) d x \leq 1\right\} .
$$

Similar with weighted Orlicz spaces, for a Young function $\Phi$ and a weight $u$ on $\mathbb{R}^{n}$, the weighted weak Orlicz space $w L_{\Phi}^{u}\left(\mathbb{R}^{n}\right)$ is the set of all measurable functions $f: \mathbb{R}^{n} \rightarrow \mathbb{R}$ such that $\|f\|_{w L_{\Phi}^{u}\left(\mathbb{R}^{n}\right)}:=\|u f\|_{w L_{\Phi}\left(\mathbb{R}^{n}\right)}<\infty$.

For $u_{1}, u_{2}: \mathbb{R}^{n} \rightarrow(0, \infty)$, we denote $u_{1} \preceq u_{2}$ if there exists a constant $C>0$ such that $u_{1}(x) \leq C u_{2}(x)$ for all $x \in \mathbb{R}^{n}$. Note that, if $u_{1} \preceq u_{2}$, then $\|f\|_{L_{\Phi}^{u_{1}}\left(\mathbb{R}^{n}\right)} \leq C\|f\|_{L_{\Phi}^{u_{2}}\left(\mathbb{R}^{n}\right)}$ and $\|f\|_{w L_{\Phi}^{u_{1}}\left(\mathbb{R}^{n}\right)} \leq C\|f\|_{w L_{\Phi}^{u_{2}}\left(\mathbb{R}^{n}\right)}$.

The study of Lebesgue spaces and Orlicz spaces has been carried out by many researchers in the last few decades (see [3, 5, 6, 7, 12, 15], etc.). In 1989, Maligranda [6] discussed inclusion properties of Orlicz spaces. Later in 2016, Masta et al. [7] obtained sufficient and necessary conditions for the inclusion relation between two Orlicz spaces and between two weak Orlicz spaces by using a different technique from Maligranda. Moreover, they have found that two Orlicz spaces and two weak Orlicz spaces are comparable with respect to Young functions for any measurable set, although the Lebesgue space $L_{p}$ are not comparable with respect to the number $p$.

On the other hand, Osançliol [13] has obtained sufficient and necessary conditions for the inclusion relation between two weighted Orlicz spaces, as in the following theorem.

Theorem 1.1. [13] Let $\Phi$ be a continuous Young function satisfying the $\triangle_{2}$ condition [that is, there exists $K>0$ such that $\Phi(2 t) \leq K \Phi(t)$ for all $t \geq 0$ ], and $u_{1}, u_{2}$ are measurable functions such that $u_{i}(x+y) \leq u_{i}(x) \cdot u_{i}(y)$ for every $x, y \in \mathbb{R}^{n}$, where $i=1,2$. Then the following statements are equivalent:

(1) $u_{1} \preceq u_{2}$.

(2) $L_{\Phi}^{u_{2}}\left(\mathbb{R}^{n}\right) \subseteq L_{\Phi}^{u_{1}}\left(\mathbb{R}^{n}\right)$.

(3) There exists a constant $C>0$ such that $\|f\|_{L_{\Phi}^{u_{1}}\left(\mathbb{R}^{n}\right)} \leq C\|f\|_{L_{\Phi}^{u_{2}}\left(\mathbb{R}^{n}\right)}$, for every $f \in L_{\Phi}^{u_{2}}\left(\mathbb{R}^{n}\right)$.

Related results for weak type of Orlicz spaces can be found in [10].

In this paper, we are interested in studying the inclusion properties of weighted Orlicz spaces. In connection with Theorem 1.1, we shall prove the inclusion relation between weighted Orlicz spaces with respect to Young functions $\Phi_{1}, \Phi_{2}$ and weights $u_{1}, u_{2}$.

To achieve our purpose, we will use the similar methods as in $[1,7,8,9,13]$, which pay attention to the characteristic functions of open balls in $\mathbb{R}^{n}$. Next, we recall some lemmas which will be used later in the next section. 
Lemma 1.2. [11] Suppose that $\Phi$ is a Young function and $\Phi^{-1}(s):=\inf \{r \geq 0$ : $\Phi(r)>s\}$. We have

(1) $\Phi^{-1}(0)=0$.

(2) $\Phi^{-1}\left(s_{1}\right) \leq \Phi^{-1}\left(s_{2}\right)$ for $s_{1} \leq s_{2}$.

(3) $\Phi\left(\Phi^{-1}(s)\right) \leq s \leq \Phi^{-1}(\Phi(s))$ for $0 \leq s<\infty$.

Lemma 1.3. [9] Let $\Phi_{1}, \Phi_{2}$ be Young functions. For any $s>0$, if there exists $C_{1}, C_{2}>0$ such that $\Phi_{2}^{-1}(s) \leq C_{1} \Phi_{1}^{-1}\left(C_{2} s\right)$, then we have $\Phi_{1}\left(\frac{t}{C_{1}}\right) \leq C_{2} \Phi_{2}(t)$ for $t=\Phi_{2}^{-1}(s)$.

In this paper, the letter $C$ will be used for constants whose values may change from line to line, while constants with subscripts, such as $C_{1}, C_{2}$, do not change their values.

\section{RESULTS}

First, we will investigate the inclusion properties of weighted Orlicz spaces with respect to distinct Young functions $\Phi_{1}$ and $\Phi_{2}$. To get the result, we need to estimate the norm of the characteristic function of an open ball in $\mathbb{R}^{n}$ as in the following lemma.

Lemma 2.1. [4, 7] Let $\Phi$ be a Young function, $a \in \mathbb{R}^{n}$, and $r>0$ be arbitrary. Then we have $\left\|\frac{\chi_{B(a, r)}}{u}\right\|_{L_{\Phi}^{u}\left(\mathbb{R}^{n}\right)}=\left\|\chi_{B(a, r)}\right\|_{E_{\Phi}\left(\mathbb{R}^{n}\right)}=\frac{1}{\Phi^{-1}\left(\frac{1}{B(a, r) \mid}\right)}$ where $|B(a, r)|$ denotes the volume of the open ball $B(a, r)$ centered at $a \in \mathbb{R}^{n}$ with radius $r>0$.

Now we come to the inclusion relation between $L_{\Phi_{1}}^{u}\left(\mathbb{R}^{n}\right)$ and $L_{\Phi_{2}}^{u}\left(\mathbb{R}^{n}\right)$ with respect to Young functions $\Phi_{1}, \Phi_{2}$. Given two Young functions $\Phi_{1}, \Phi_{2}$, we write $\Phi_{1} \prec \Phi_{2}$ if there exists a constant $C>0$ such that $\Phi_{1}(t) \leq \Phi_{2}(C t)$ for all $t>0$.

Theorem 2.2. Let $\Phi_{1}, \Phi_{2}$ be Young functions and $u: \mathbb{R}^{n} \rightarrow(0, \infty)$ be a measurable function. Then the following statements are equivalent:

(1) $\Phi_{1} \prec \Phi_{2}$.

(2) $L_{\Phi_{2}}^{u}\left(\mathbb{R}^{n}\right) \subseteq L_{\Phi_{1}}^{u}\left(\mathbb{R}^{n}\right)$

(3) There exists a constant $C>0$ such that $\|f\|_{L_{\Phi_{1}}^{u}\left(\mathbb{R}^{n}\right)} \leq C\|f\|_{L_{\Phi_{2}}^{u}\left(\mathbb{R}^{n}\right)}$, for every $f \in L_{\Phi_{2}}^{u}\left(\mathbb{R}^{n}\right)$.

Proof. Assume that (1) holds. Suppose that $f \in L_{\Phi_{2}}^{u}\left(\mathbb{R}^{n}\right)$. Observe that

$$
\begin{aligned}
\int_{\mathbb{R}^{n}} \Phi_{1}\left(\frac{|u(x) f(x)|}{C\|f\|_{L_{\Phi_{2}}^{u}\left(\mathbb{R}^{n}\right)}}\right) d x & \leq \int_{\mathbb{R}^{n}} \Phi_{2}\left(\frac{C|u(x) f(x)|}{C\|f\|_{L_{\Phi_{2}}^{u}\left(\mathbb{R}^{n}\right)}}\right) d x \\
& =\int_{\mathbb{R}^{n}} \Phi_{2}\left(\frac{|u(x) f(x)|}{\|f\|_{L_{\Phi_{2}}^{u}\left(\mathbb{R}^{n}\right)}}\right) d x \leq 1 .
\end{aligned}
$$


By definition of $\|\cdot\|_{L_{\Phi}^{u}\left(\mathbb{R}^{n}\right)}$, we have $\|f\|_{L_{\Phi_{1}}^{u}\left(\mathbb{R}^{n}\right)} \leq C\|f\|_{L_{\Phi_{2}}^{u}\left(\mathbb{R}^{n}\right)}$. This proves that $L_{\Phi_{2}}^{u}\left(\mathbb{R}^{n}\right) \subseteq L_{\Phi_{1}}^{u}\left(\mathbb{R}^{n}\right)$.

Next, since $\left(L_{\Phi_{1}}^{u}\left(\mathbb{R}^{n}\right), L_{\Phi_{2}}^{u}\left(\mathbb{R}^{n}\right)\right)$ is a Banach pair, it follows from [2, Lemma $3.3]$ that (2) and (3) are equivalent. It thus remains to show that (3) implies (1).

Assume now that (3) holds. By Lemma 2.1, we have

$$
\frac{1}{\Phi_{1}^{-1}\left(\frac{1}{|B(a, r)|}\right)}=\left\|\frac{\chi_{B(a, r)}}{u}\right\|_{L_{\Phi_{1}}^{u}\left(\mathbb{R}^{n}\right)} \leq C\left\|\frac{\chi_{B(a, r)}}{u}\right\|_{L_{\Phi_{2}}^{u}\left(\mathbb{R}^{n}\right)}=\frac{C}{\Phi_{2}^{-1}\left(\frac{1}{|B(a, r)|}\right)} .
$$

Since $\frac{1}{\Phi_{1}^{-1}\left(\frac{1}{|B(a, r)|}\right)} \leq \frac{C}{\Phi_{2}^{-1}\left(\frac{1}{|B(a, r)|}\right)}$ is equivalent to $C \Phi_{1}^{-1}\left(\frac{1}{|B(a, r)|}\right) \geq \Phi_{2}^{-1}\left(\frac{1}{|B(a, r)|}\right)$ for arbitrary $a \in \mathbb{R}^{n}$ and $r>0$, by Lemma 1.3 , we have

$$
\Phi_{1}\left(\frac{t_{0}}{C}\right) \leq \Phi_{2}\left(t_{0}\right)
$$

for $t_{0}=\Phi_{2}^{-1}\left(\frac{1}{|B(a, r)|}\right)$. Since $a \in \mathbb{R}^{n}$ and $r>0$ are arbitrary, we conclude that $\Phi_{1}(t) \leq \Phi_{2}(C t)$ for every $t>0$.

Remark 2.3. For $u(x)=1$, Theorem 2.2 reduces to Theorem 2.5 in [7].

Next, we also give the sufficient and necessary conditions for inclusion relation between weighted Orlicz spaces $L_{\Phi_{1}}^{u_{1}}\left(\mathbb{R}^{n}\right)$ and $L_{\Phi_{2}}^{u_{2}}\left(\mathbb{R}^{n}\right)$ with respect to Young functions $\Phi_{1}, \Phi_{2}$ and weights $u_{1}, u_{2}$. To get the result, we need the following lemma.

Lemma 2.4. Let $u: \mathbb{R}^{n} \rightarrow(0, \infty)$ be a measurable function such that $u(x+y) \leq$ $u(x) \cdot u(y)$ for every $x, y \in \mathbb{R}^{n}$. If $\Phi$ is a Young function, then:

(1) For all $f \in L_{\Phi}^{u}\left(\mathbb{R}^{n}\right)$ and for all $x \in \mathbb{R}^{n}$, we have $\left\|T_{x} f\right\|_{L_{\Phi}^{u}\left(\mathbb{R}^{n}\right)} \leq u(x)\|f\|_{L_{\Phi}^{u}\left(\mathbb{R}^{n}\right)}$, where $T_{x} f(y)=f(y-x)$.

(2) If $f \in L_{\Phi}^{u}\left(\mathbb{R}^{n}\right)$ and $f \neq 0$, then there exists a constant $C>0$ (depending on $f$ ) such that

$$
\frac{u(x)}{C} \leq\left\|T_{x} f\right\|_{L_{\Phi}^{u}\left(\mathbb{R}^{n}\right)} \leq C u(x) .
$$

Proof.

(1) Let $f \in L_{\Phi}^{u}\left(\mathbb{R}^{n}\right)$ and $T_{x} f(y)=f(y-x)$. Then

$$
\int_{\mathbb{R}^{n}} \Phi\left(\frac{|u(v) f(v)|}{\|f\|_{L_{\Phi}^{u}\left(\mathbb{R}^{n}\right)}}\right) d v \leq 1
$$


Observe that (by setting $v:=y-x$ ), we have

$$
\begin{aligned}
\int_{\mathbb{R}^{n}} \Phi\left(\frac{\left|u(y) T_{x} f(y)\right|}{u(x)\|f\|_{L_{\Phi}^{u}\left(\mathbb{R}^{n}\right)}}\right) d y & =\int_{\mathbb{R}^{n}} \Phi\left(\frac{|u(y) f(y-x)|}{u(x)\|f\|_{L_{\Phi}^{u}\left(\mathbb{R}^{n}\right)}}\right) d y \\
& =\int_{\mathbb{R}^{n}} \Phi\left(\frac{|u(v+x) f(v)|}{u(x)\|f\|_{L_{\Phi}^{u}\left(\mathbb{R}^{n}\right)}}\right) d v \\
& \leq \int_{\mathbb{R}^{n}} \Phi\left(\frac{|u(v) u(x) f(v)|}{u(x)\|f\|_{L_{\Phi}^{u}\left(\mathbb{R}^{n}\right)}}\right) d v \\
& =\int_{\mathbb{R}^{n}} \Phi\left(\frac{|u(v) f(v)|}{\|f\|_{L_{\Phi}^{u}\left(\mathbb{R}^{n}\right)}}\right) d v \\
& \leq 1
\end{aligned}
$$

This shows that $\left\|T_{x} f\right\|_{L_{\Phi}^{u}\left(\mathbb{R}^{n}\right)} \leq u(x)\|f\|_{L_{\Phi}^{u}\left(\mathbb{R}^{n}\right)}$.

(2) Let $f \in L_{\Phi}^{u}\left(\mathbb{R}^{n}\right)$ and $f \neq 0$. Then there exists a constant $C>0$ (depending on $f$ ) such that $\|f\|_{L_{\Phi}^{u}\left(\mathbb{R}^{n}\right)} \leq C$. By Lemma $2.4(1)$, we have

$$
\left\|T_{x} f\right\|_{L_{\phi, \Phi}^{u}\left(\mathbb{R}^{n}\right)} \leq C u(x)
$$

for every $x \in \mathbb{R}^{n}$. Since $f(x) \neq 0$ for every $x \in \mathbb{R}^{n}$, we have $\left\|T_{x} f\right\|_{L_{\Phi}^{u}\left(\mathbb{R}^{n}\right)}>0$. Observe that

$$
\begin{aligned}
& \int_{\mathbb{R}^{n}} \Phi\left(\frac{|u(x) f(v)|}{\sup _{v \in \mathbb{R}^{n}} u(-v)\left\|T_{x} f\right\|_{L_{\Phi}^{u}\left(\mathbb{R}^{n}\right)}}\right) d v \leq \int_{\mathbb{R}^{n}} \Phi\left(\frac{|u(x) f(v)|}{u(-v)\left\|T_{x} f\right\|_{L_{\Phi}^{u}\left(\mathbb{R}^{n}\right)}}\right) d v \\
& \leq \int_{\mathbb{R}^{n}} \Phi\left(\frac{|u(v+x) f(v)|}{\left\|T_{x} f\right\|_{L_{\Phi}^{u}\left(\mathbb{R}^{n}\right)}}\right) d v \\
& \leq \int_{\mathbb{R}^{n}} \Phi\left(\frac{|u(y) f(y-x)|}{\left\|T_{x} f\right\|_{L_{\Phi}^{u}\left(\mathbb{R}^{n}\right)}}\right) d y \\
& =\int_{\mathbb{R}^{n}} \Phi\left(\frac{\left|u(y) L_{x} f(y)\right|}{\left\|T_{x} f\right\|_{L_{\Phi}^{u}\left(\mathbb{R}^{n}\right)}}\right) d y \\
& \leq 1 \text {. }
\end{aligned}
$$

This shows that $\frac{u(x)\|f\|_{L_{\Phi}\left(\mathbb{R}^{n}\right)}}{\sup _{v \in R^{n}} u(-v)} \leq\left\|T_{x} f\right\|_{L_{\Phi}^{u}\left(\mathbb{R}^{n}\right)}$.

Choose $C:=\max \left\{C_{1}, \frac{\sup _{v \in \mathbb{R}^{n}} u(-v)}{\|f\|_{L_{\Phi}\left(\mathbb{R}^{n}\right)}}\right\}$. Hence, we conclude that

$$
\frac{u(x)}{C} \leq\left\|T_{x} f\right\|_{L_{\Phi}^{u}\left(\mathbb{R}^{n}\right)} \leq C u(x)
$$

as desired.

Now, we present the sufficient and necessary conditions for the inclusion relation between weighted Orlicz spaces $L_{\Phi_{1}}^{u_{1}}\left(\mathbb{R}^{n}\right)$ and $L_{\Phi_{2}}^{u_{2}}\left(\mathbb{R}^{n}\right)$ with respect to Young functions $\Phi_{1}, \Phi_{2}$ and weights $u_{1}, u_{2}$. 
Theorem 2.5. Let $\Phi_{1}, \Phi_{2}$ be Young functions such that $\Phi_{1} \prec \Phi_{2}$ and $u_{1}, u_{2}$ are measurable functions such that $u_{i}(x+y) \leq u_{i}(x) \cdot u_{i}(y)$ for every $x, y \in \mathbb{R}^{n}$, where $i=1,2$. Then the following statements are equivalent:

(1) $u_{1} \preceq u_{2}$.

(2) $L_{\Phi_{2}}^{u_{2}}\left(\mathbb{R}^{n}\right) \subseteq L_{\Phi_{1}}^{u_{1}}\left(\mathbb{R}^{n}\right)$.

(3) There exists a constant $C>0$ such that $\|f\|_{L_{\Phi_{1}}^{u_{1}}\left(\mathbb{R}^{n}\right)} \leq C\|f\|_{L_{\Phi_{2}}^{u_{2}\left(\mathbb{R}^{n}\right)}}$, for every $f \in L_{\Phi_{2}}^{u_{2}}\left(\mathbb{R}^{n}\right)$.

Proof.

Assume that (1) holds. Let $f$ be an element of $L_{\Phi_{2}}^{u_{2}}\left(\mathbb{R}^{n}\right)$. Since $\Phi_{1} \prec \Phi_{2}$ and $u_{1} \preceq u_{2}$, there exists constants $C_{1}, C_{2}>0$ such that $\Phi_{1}(t) \leq \Phi_{2}\left(C_{1} t\right)$ for all $t>0$ and $u_{1}(x) \leq C_{2} u_{2}(x)$ for every $x \in \mathbb{R}^{n}$. Using a similar argument as in the proof of Theorem 2.2 we have

$$
\|f\|_{L_{\Phi_{1}}^{u_{1}}\left(\mathbb{R}^{n}\right)} \leq C_{1}\|f\|_{L_{\Phi_{2}}^{u_{1}}\left(\mathbb{R}^{n}\right)} \leq C_{1} C_{2}\|f\|_{L_{\Phi_{2}}^{u_{2}}\left(\mathbb{R}^{n}\right)} .
$$

As before, we have that (2) and (3) are equivalent. It thus remains to show that (3) implies (1). Assume that (3) holds. By Lemma 2.4, we have

$$
\frac{u_{1}(x)}{C} \leq\left\|T_{x} f\right\|_{L_{\Phi_{1}}^{u_{1}}\left(\mathbb{R}^{n}\right)} \leq C\left\|T_{x} f\right\|_{L_{\Phi_{2}}^{u_{2}\left(\mathbb{R}^{n}\right)}} \leq C u_{2}(x),
$$

for every $x \in \mathbb{R}^{n}$. So, we obtain $u_{1} \preceq u_{2}$.

Note that, for $\Phi_{1}(x)=\Phi_{2}(x)$ for every $x \in \mathbb{R}^{n}$, Theorem 2.5 reduces to Theorem 1.1.

Remark 2.6. It follows from Theorems 2.2 and 2.5 that there cannot be an inclusion relation between $L_{p_{1}}^{u_{1}}\left(\mathbb{R}^{n}\right)$ and $L_{p_{2}}^{u_{2}}\left(\mathbb{R}^{n}\right)$ for distinct values of $p_{1}$ and $p_{2}$. In spite of that, for finite measure set $X$ we can obtain an inclusion relation between $L_{p_{1}}^{u_{1}}(X)$ and $L_{p_{2}}^{u_{2}}(X)$ as presented in the next section.

\section{AN ADDITIONAL CASE}

In the following, we will give a sufficient condition for Hölder's inequality in weighted Orlicz spaces which will be used to obtain an inclusion relation between $L_{p_{1}}^{u_{1}}(X)$ and $L_{p_{2}}^{u_{2}}(X)$.

Theorem 3.1. (Hölder's inequality) Let $X$ be a measurable set, $\Phi_{1}, \Phi_{2}, \Phi_{3}$ be Young functions and $u_{1}, u_{2}, u_{3}: X \rightarrow \mathbb{R}$ be measurable functions such that

$$
\Phi_{1}^{-1}(t) \Phi_{2}^{-1}(t) \leq \Phi_{3}^{-1}(t)
$$

for every $t>0$ and $u_{3}(x) \leq u_{1}(x) u_{2}(x)$ for every $x \in X$. If $f_{1} \in L_{\Phi_{1}}^{u_{1}}(X)$ and $f_{2} \in L_{\Phi_{2}}^{u_{2}}(X)$, then $f_{1} f_{2} \in L_{\Phi_{3}}^{u_{3}}(X)$ with

$$
\left\|f_{1} f_{2}\right\|_{L_{\Phi_{3}}^{u_{3}(X)}} \leq 2\left\|f_{1}\right\|_{L_{\Phi_{1}}^{u_{1}}(X)}\left\|f_{2}\right\|_{L_{\Phi_{2}}^{u_{2}(X)}} .
$$


Proof. Let $s, t \geq 0$. Without loss of generality, suppose that $\Phi_{1}(s) \leq \Phi_{2}(t)$. By Lemma 1.2(3), we obtain

$$
s t \leq \Phi_{1}^{-1}\left(\Phi_{1}(s)\right) \Phi_{2}^{-1}\left(\Phi_{2}(t)\right) \leq \Phi_{1}^{-1}\left(\Phi_{2}(t)\right) \Phi_{2}^{-1}\left(\Phi_{2}(t)\right) \leq \Phi_{3}^{-1}\left(\Phi_{2}(t)\right) .
$$

Hence $\Phi_{3}(s t) \leq \Phi_{3}\left(\Phi_{3}^{-1}\left(\Phi_{2}(t)\right)\right) \leq \Phi_{2}(t) \leq \Phi_{2}(t)+\Phi_{1}(s)$. Since $\Phi$ is a convex function, we have

$$
\begin{aligned}
\int_{X} \Phi_{3}\left(\frac{\left|u_{3}(x) f_{1}(x) f_{2}(x)\right|}{2\left\|f_{1}\right\|_{L_{\Phi_{1}}^{u_{1}}(X)}\left\|f_{2}\right\|_{L_{\Phi_{2}}^{u_{2}(X)}}}\right) d x & \leq \frac{1}{2} \int_{X} \Phi_{3}\left(\frac{\left|u_{3}(x) f_{1}(x) f_{2}(x)\right|}{\left\|f_{1}\right\|_{L_{\Phi_{1}}^{u_{1}}(X)}\left\|f_{2}\right\|_{L_{\Phi_{2}}^{u_{2}(X)}}}\right) d x \\
& \leq \frac{1}{2} \int_{X} \Phi_{3}\left(\frac{\left|u_{1}(x) u_{2}(x) f_{1}(x) f_{2}(x)\right|}{\left\|f_{1}\right\|_{L_{\Phi_{1}}^{u_{1}}(X)}\left\|f_{2}\right\|_{L_{\Phi_{2}}^{u_{2}}(X)}}\right) d x .
\end{aligned}
$$

On the other hand, by Lemma 2.1, we obtain

$$
\begin{aligned}
\int_{X} \Phi_{3}\left(\frac{\left|u_{1}(x) u_{2}(x) f_{1}(x) f_{2}(x)\right|}{\left\|f_{1}\right\|_{L_{\Phi_{1}}^{u_{1}}(X)}\left\|f_{2}\right\|_{L_{\Phi_{2}}^{u_{2}(X)}}}\right) d x \leq & \int_{X} \Phi_{1}\left(\frac{\left|u_{1}(x) f_{1}(x)\right|}{\left\|f_{1}\right\|_{L_{\Phi_{1}}^{u_{1}}(X)}}\right) d x \\
& +\int_{X} \Phi_{2}\left(\frac{\left|f_{2}(x)\right|}{\left\|f_{2}\right\|_{L_{\Phi_{2}}^{u_{2}}(X)}}\right) d x \leq 2
\end{aligned}
$$

whenever $f_{1} \in L_{\Phi_{1}}^{u_{1}}(X)$ and $f_{2} \in L_{\Phi_{2}}^{u_{2}}(X)$. By the definition of $\|\cdot\|_{L_{\Phi_{3}}^{u_{1}}(X)}$, we have $\left\|f_{1} f_{2}\right\|_{L_{\Phi_{3}}^{u_{3}(X)}} \leq 2\left\|f_{1}\right\|_{L_{\Phi_{1}}^{u_{1}(X)}}\left\|f_{2}\right\|_{L_{\Phi_{2}}^{u_{2}(X)}}$, as desired.

Corollary 3.2. Let $X:=B\left(a, r_{0}\right) \subseteq \mathbb{R}^{n}$ for some $a \in \mathbb{R}^{n}$ and $r_{0}>0$. If $\Phi_{1}, \Phi_{2}$ are two Young functions, $u_{1}, u_{2}: X \rightarrow \mathbb{R}$ are measurable functions and there exist a Young function $\Phi$ and a weight $0<u(x) \leq 1$ for every $x \in X$ such that $\Phi_{1}^{-1}(t) \Phi^{-1}(t) \leq \Phi_{2}^{-1}(t)$ for every $t \geq 0$ and $u_{1}(x) \leq u(x) u_{2}(x)$ for every $x \in X$, then

$$
L_{\Phi_{1}}^{u_{1}}(X) \subseteq L_{\Phi_{2}}^{u_{2}}(X)
$$

with $\|f\|_{L_{\Phi_{2}}^{u_{2}(X)}} \leq \frac{2}{\Phi^{-1}\left(\frac{1}{\mid B\left(a, r_{0}\right)}\right)}\|f\|_{L_{\Phi_{1}}^{u_{1}}(X)}$ for $f \in L_{\Phi_{1}}^{u_{1}}(X)$.

Proof. Since $0<u(x) \leq 1$ for every $x \in X$, we have $\|f\|_{L_{\Phi_{2}}^{u_{2}(X)}} \leq\left\|\frac{f}{u}\right\|_{L_{\Phi_{2}}^{u_{2}(X)}}$. Let $f \in L_{\Phi_{1}}^{u_{1}}(X)$. By Theorem 3.1 and choosing $g:=\chi_{B\left(a, r_{0}\right)}$, we obtain

$$
\begin{aligned}
\|f\|_{L_{\Phi_{2}}^{u_{2}(X)}} & =\left\|f \chi_{B\left(a, r_{0}\right)}\right\|_{L_{\Phi_{2}}^{u_{2}(X)}} \\
& =\|f g\|_{L_{\Phi_{2}}^{u_{2}(X)}} \\
& \leq\left\|\frac{f g}{u}\right\|_{L_{\Phi_{2}}^{u_{2}(X)}} \\
& \leq 2\left\|\frac{g}{u}\right\|_{L_{\Phi}^{u}(X)}\|f\|_{L_{\Phi_{1}}^{u_{1}}(X)} \\
& =\frac{2}{\Phi^{-1}\left(\frac{1}{\left|B\left(a, r_{0}\right)\right|}\right)}\|f\|_{L_{\Phi_{1}}^{u_{1}}(X)} .
\end{aligned}
$$


This shows that $L_{\Phi_{1}}^{u_{1}}(X) \subseteq L_{\Phi_{2}}^{u_{2}}(X)$.

We shall now discuss the inclusion properties of weighted weak Lebesgue spaces $L_{p_{1}}^{u_{1}}(X)$ and $L_{p_{2}}^{u_{2}}(X)$ with respect to distinct values of $p_{1}$ and $p_{2}$ as well as $u_{1}$ and $u_{2}$.

Corollary 3.3. Let $X:=B\left(a, r_{0}\right)$ for some $a \in \mathbb{R}^{n}$ and $r_{0}>0$. If $1 \leq p_{2}<p_{1}<\infty$ and $u_{1}, u_{2}: X \rightarrow \mathbb{R}$ are measurable functions such that $u_{1}(x) \leq u_{2}(x)$ for every $x \in X$, then

$$
L_{p_{1}}^{u_{1}}(X) \subseteq L_{p_{2}}^{u_{2}}(X) .
$$

Proof. Let $\Phi_{1}(t):=t^{p_{1}}, \Phi_{2}(t):=t^{p_{2}}$, and $\Phi(t):=t^{\frac{p_{1} p_{2}}{p_{1}-p_{2}}}$ for every $t \geq 0$. Since $1 \leq p_{2}<p_{1}<\infty$, we have $\frac{p_{1} p_{2}}{p_{1}-p_{2}}>1$. Thus, $\Phi_{1}, \Phi_{2}$, and $\Phi$ are Young functions. Now, define $u(x)=\frac{u_{1}(x)}{u_{2}(x)}$ for every $x \in X$. Observe that, using the definition of $\Phi^{-1}$ and Lemma 1.2, we have

$$
\Phi_{1}^{-1}(t)=t^{\frac{1}{p_{1}}}, \Phi_{2}^{-1}(t)=t^{\frac{1}{p_{2}}}, \text { and } \Phi^{-1}(t)=t^{\frac{p_{1}-p_{2}}{p_{1} p_{2}}} .
$$

Moreover, $\Phi_{1}^{-1}(t) \Phi^{-1}(t)=t^{\frac{1}{p_{1}}} t^{\frac{p_{1}-p_{2}}{p_{1} p_{2}}}=t^{\frac{1}{p_{2}}}=\Phi_{2}^{-1}(t)$ and $u_{1}(x)=\frac{u_{1}(x)}{u_{2}(x)} u_{2}(x)=$ $u(x) u_{2}(x)$. So it follows from Corollary 3.2 that

$$
\|f\|_{L_{p_{2}}^{u_{2}}(X)} \leq 2\left|B\left(a, r_{0}\right)\right|^{\frac{p_{1}-p_{2}}{p_{1} p_{2}}}\|f\|_{L_{p_{1}}^{u_{1}(X)}},
$$

and therefore we can conclude that $L_{p_{1}}^{u_{1}}(X) \subseteq L_{p_{2}}^{u_{2}}(X)$.

\section{CONCLUDING REMARKS}

We have shown the inclusion properties of weighted Orlicz spaces for distinct Young functions $\Phi_{1}, \Phi_{2}$ and weights $u_{1}, u_{2}$. The inclusion properties of weighted Orlicz spaces are a generalization of inclusion properties of Orlicz spaces in [7] and inclusion properties of weighted Lebesgue spaces. In the proof of our results, we used the norm of characteristic function on $\mathbb{R}^{n}$ and estimated the norm of the translation functions in $\mathbb{R}^{n}$.

Furthemore, from Theorem 2.5 and Lemma 1.1, Theorem 2.8 in [10], we also have the following inclusion relations

$$
\begin{array}{ccc}
L_{\Phi_{2}}^{u_{2}} & \rightarrow & L_{\Phi_{1}}^{u_{1}} \\
\downarrow & \searrow & \downarrow \\
w L_{\Phi_{2}}^{u_{2}} & \rightarrow & w L_{\Phi_{1}}^{u_{1}}
\end{array}
$$

for $\Phi_{1} \prec \Phi_{2}$ and $u_{1} \preceq u_{2}$, where the arrows mean 'contained in' or 'embedded into'.

Acknowledgement. The first author is supported by Hibah Penguatan Kompetensi UPI 2018. The authors thank the referee for his/her useful remarks on the earlier version of this paper. 


\section{REFERENCES}

[1] H. Gunawan, D.I. Hakim, K.M. Limanta, and A.A. Masta, "Inclusion properties of generalized Morrey spaces", Math. Nachr. 290 (2017), 332-340. [DOI: 10.1002/mana.201500425]

[2] S.G. Kreín, Yu.I. Petunīn, and E.M. Semënov, Interpolation of Linear Operators, Translation of Mathematical Monograph vol. 54, American Mathematical Society, Providence, R.I., 1982.

[3] A. Kufner, O. John, and S. Fucik, Function Spaces, Noordhoff International Publishing, Czechoslovakia, 1977.

[4] N. Liu and Y. Ye, "Weak Orlicz space and its convergence theorems", Acta Math. Sci. Ser. B 30-5 (2010), 1492-1500.

5] W.A.J. Luxemburg, Banach Function Spaces, Thesis, Technische Hogeschool te Delft, 1955.

[6] L. Maligranda, Orlicz Spaces and Interpolation, Departamento de Matemática, Universidade Estadual de Campinas, 1989.

[7] A.A. Masta, H. Gunawan, and W. Setya-Budhi, "Inclusion property of Orlicz and weak Orlicz spaces", J. Math. Fund. Sci. 48-3 (2016), 193-203 [DOI: http://dx.doi.org/10.56142Fj.math.fund.sci.2016.48.3.1].

[8] A.A. Masta, H. Gunawan, and W. Setya-Budhi, "An inclusion property of Orlicz-Morrey spaces", J. Phys.: Conf. Ser., 893012015 (2017), 1-8 [DOI: https://doi.org/10.1088/17426596/893/1/012015].

[9] A.A. Masta, H. Gunawan, and W. Setya-Budhi, "On inclusion properties of two versions of Orlicz-Morrey spaces", Mediterr. J. Math., 14-6 (2017), 228-239. [DOI: https://doi.org/10.1007/s00009-017-1030-7]

[10] A.A. Masta, Ifronika, and M. Taqiyuddin, "Inclusion properties of weighted weak Orlicz spaces", research report (https://arxiv.org/abs/1710.04537), 2017.

[11] E. Nakai, "On Orlicz-Morrey spaces", Research Report [http://repository.kulib.kyotou.ac.jp/dspace/bitstream/2433/58769/1/1520- 10.pdf, accessed on August 17, 2015.]

[12] W. Orlicz, Linear Functional Analysis (Series in Real Analysis Volume 4), World Scientific, Singapore, 1992.

[13] A. Osançliol, "Inclusion between weighted Orlicz spaces", J. Inequal. Appl. 2014-390 (2014), 1-8 [DOI: https://doi.org/10.1186/1029-242X-2014-390].

[14] M.M. Rao and Z.D. Ren, Theory of Orlicz Spaces, Monographs and Textbooks in Pure and Applied Mathematics Vol. 146, Marcel Dekker, Inc., New York, 1991.

[15] M. Taqiyuddin and A.A. Masta, "Inclusion properties of Orlicz spaces and weak Orlicz spaces generated by concave function", IOP Conf. Ser.: Mater. Sci. Eng., 288012103 (2018), 1-5 [DOI: https://doi.org/10.1088/1757-899X/288/1/012103].

[16] X. Zhang and C. Zhang, "Weak Orlicz spaces generated by concave functions", International Conference on Information Science and Technology (ICIST) 2011, 42-44. 